\title{
On Automorphisms of Transformation Semigroups
}

\author{
by \\ Inessa Levi \\ Department of Mathematics, University of Louisville, \\ Louisville, U.S.A. \\ and \\ Graham Wood \\ Department of Mathematics and Statistics \\ University of Canterbury, Christchurch, New Zealand.
}

No. 87

May, 1993 


\title{
On Automorphisms of Transformation Semigroups
}

\author{
Inessa Levi and G.R. Wood \\ Communicated by Boris M. Schein
}

This paper addresses the problem of describing automorphisms of semigroups of transformations. In [2] we were involved in characterizing all automorphisms of CroisotTeissier semigroups. The semigroups of transformations that belong to this large family generally consist of many-to-one transformations whose restrictions to range sets are oneto-one. Here we consider enlargements of Croisot-Teissier semigroups whose elements, restricted to range-sets, are no longer one-to-one. We show that such semigroups contain a maximal Croisot-Teissier semigroup, which in turn is used to present a complete description of automorphisms of these semigroups. Moreover we describe the Green's relations on these enlargements of Croisot-Teissier semigroups, and show that they are in fact simple semigroups, whose regular elements form a bisimple subsemigroup. We start by recalling the definition of Croisot-Teissier semigroups.

Let $p$ and $q$ be infinite cardinals with $p \geq q$, and let $X$ be a set with $|X| \geq p$. Let $\mathcal{E}=\left\{\mathcal{E}_{i} \mid i \in I\right\}$ be a set of distinct equivalences on $X$ such that $\left|X / \mathcal{E}_{i}\right|=p$ for all $i \in I$. A subset $A$ of $X$ is said to be well-separated (w.s.) by $\mathcal{E}$ if $|A|=p$ and $\mathcal{E}_{i} \cap(A \times A)$ is the identity relation on $A$ for all $i \in I$. For a cardinal $t$, with $q \leq t \leq p$, let $\mathcal{C}_{t}=\{$ w.s. $A \mid$ for some w.s. $B, A \subseteq B$ and $|B-A|=t\}$. When $X$ contains a w.s. set, the Croisot-Teissier semigroup on $X, \mathcal{E}$ of type $(p, q)$ is $C T(X, \mathcal{E}, p, q)=$ $\left\{f: X \rightarrow X \mid \pi(f) \in \mathcal{E}, R(f) \in \mathcal{C}_{q}\right\}$ with the operation of function composition [1]. Recall that for a transformation $f$ of $X, R(f)=f(X)$ denotes the range of $f$, and $\pi(f)$ denotes the partition of $X$ determined by $f$ such that $x$ and $y$ are in the same class of $\pi(f)$ if and only if $f(x)=f(y)$.

A Croisot-Teissier semigroup $C T(X, \mathcal{E}, p, q)$ is idempotent-free and either simple (when $p=q$ ) or has a minimal ideal $C T(X, \mathcal{E}, p, p)$ that itself is a Croisot-Teissier semigroup. A simple Croisot-Teissier semigroup $C T(X, \mathcal{E}, p, p)$ is the disjoint union of its minimal left ideals, and any simple idempotent-free semigroup with a minimal left ideal can be embedded in a simple Croisot-Teissier semigroup $C T(X, \mathcal{E}, p, p)$. The Green's relations on these semigroups were described in [3], and their congruences were studied in [4], [5], [6], [7] and [8]. 
We construct the following generalization of a Croisot-Teissier semigroup. In view of the intimate connection between equivalences on $X$ and partitions of $X$ we write $[x] \in \mathcal{B}$ to indicate that $[x]$ is the equivalence class of the equivalence $\mathcal{B}$ containing $x \in X$. Given an infinite cardinal $r \leq p$, and an equivalence $\mathcal{A}$ on $X$ let $\mathcal{A}^{(r)}$ be the family of all equivalences $\mathcal{B}$ on $X$ such that $\mathcal{A} \subseteq \mathcal{B}$ and for every $[x] \in \mathcal{B},|[x] / \mathcal{A}|<r$. Informally, such a $\mathcal{B}$ in $\mathcal{A}^{(r)}$ is formed by glueing together classes of $\mathcal{A}$, with each class in $\mathcal{B}$ made up of fewer than $r$ classes of $\mathcal{A}$. The family $\mathcal{A}^{(r)}$ is referred to as the family of $r$ glueings of $\mathcal{A}$. Let $\mathcal{E}^{(r)}=\cup\left\{\mathcal{E}_{i}^{(r)} \mid i \in I\right\}$ be the family of $r$ glueings of $\mathcal{E}$ and

$$
S=\left\{f: X \rightarrow X \mid R(f) \in \mathcal{C}_{q} \text { and } \pi(f) \in \mathcal{E}^{(r)}\right\}
$$

The above semigroup $S$ contains a maximal Croisot-Teissier subsemigroup $S^{\#}$ that generally does not coincide with the original $C T(X, \mathcal{E}, p, q)$. Let $\mathcal{E}^{\#}=\left\{\mathcal{A} \in \mathcal{E}^{(r)} \mid \pi(t)=\right.$ $\pi(f t)$, for all $f, t \in S$ with $\pi(f)=\mathcal{A}\}$ and let $S^{\#}=C T\left(X, \mathcal{E}^{\#}, p, q\right)$. We show that $S^{\#}$ is a subsemigroup of $S$ containing $C T(X, \mathcal{E}, p, q)$. Let $\mathcal{C}_{q}^{\#}$ be the set of ranges of all the mappings in $S^{\#}$. If $A \in \mathcal{C}_{q}$ and $\mathcal{B} \in \mathcal{E}^{\#}$ then $(A \times A) \cap \mathcal{B}=i_{A}$, else for $f, t \in S$ with $\pi(f)=\mathcal{B}$ and $R(t)=A, \pi(f t)$ and $\pi(t)$ are distinct, a contradiction. Therefore $\mathcal{C}_{q}$ is a subset of $\mathcal{C}_{q}^{\#}$. Moreover since $\mathcal{E}$ is a subset of $\mathcal{E}^{\#}, \mathcal{C}_{q}^{\#}$ is a subset of $\mathcal{C}_{q}$, and so the next result follows from the above and the observation that for any $f$ and $g$ in a Croisot-Teissier semigroup, $\pi(f g)=\pi(g)$.

Proposition $1 S^{\#}$ is a maximal Croisot-Teissier subsemigroup of $S$.

In the following example we start with a specific Croisot-Teissier semigroup and construct the associated $\mathcal{E}^{(r)}$ and $\mathcal{E}^{\#}$. The example is based on [2, Example 4.2].

Example Let $\mathbf{R}$ be the set of all real numbers, and $\mathbf{R}^{+}$be the set of all positive reals. For each $a \in \mathbf{R}^{+}$let $\mathcal{E}_{a}$ be the equivalence on $\mathbf{R}$ whose only non-singleton class is $[a]=\{a\} \cup\left(\mathbf{R}-\mathbf{R}^{+}\right)$. Let $\mathcal{E}_{0}$ be the equivalence on $\mathbf{R}$ having two non-singleton classes: $[-1]=\{b \in \mathbf{R}:-1 \leq b \leq 0\}$ and $[-2]=\{b \in \mathbf{R}: b<-1\}$. Let $\mathcal{E}=\left\{\mathcal{E}_{b}: b \in\right.$ $\mathbf{R}, b \geq 0\}$, and $p=q=|\mathbf{R}|$. Note that the $\mathcal{C}_{p}$ sets are those subsets $A$ of $\mathbf{R}^{+}$having $|A|=\left|\mathbf{R}^{+}-A\right|$, and that the semigroup $C T(\mathbf{R}, \mathcal{E}, p, p)$ consists of all transformations $f: \mathbf{R} \rightarrow \mathbf{R}$ having $\pi(f) \in \mathcal{E}$ and $R(f) \in \mathcal{C}_{p}$.

If $r=\aleph_{0}, \mathcal{E}^{(r)}$ is the set of all equivalences on $\mathbf{R}$ whose non-singleton classes are of the form $Y^{\prime} \cup Y^{\prime \prime}$ where $Y^{\prime}$ is either $[-1],[-2], \mathbf{R}-\mathbf{R}^{+}$, or empty, and $Y^{\prime \prime}$ is either a finite subset of $\mathbf{R}^{+}$or empty. Let $S=\left\{f: \mathbf{R} \rightarrow \mathbf{R} \mid R(f) \in \mathcal{C}_{p}, \pi(f) \in \mathcal{E}^{(r)}\right\}$. Since $\mathcal{E}^{\#}$ is just $\mathcal{E}$ together with all partitions in $\mathcal{E}^{(r)}$ for which every $\mathcal{C}_{p}$ set is a partial transversal, $\mathcal{E}^{\#}$ consists of all equivalences in $\mathcal{E}^{(r)}$ whose non-singleton classes are of the form $Y^{\prime} \cup Y^{\prime \prime}$, where $Y^{\prime}, Y^{\prime \prime}$ are as above with $\left|Y^{\prime \prime}\right| \leq 1$. Thus we have that $C T(\mathbf{R}, \mathcal{E}, p, p) \subset S^{\#}=$ $C T\left(X, \mathcal{E}^{\#}, p, p\right) \subset S$.

We show that the restriction $\phi^{\#}$ of an automorphism $\phi$ of $S$ to $S^{\#}$ is a rangepreserving, $r$ union-preserving and $r$ glueing-preserving automorphism of $S^{\#}$ (see Definitions 2,4 , and 5 below), and that every such automorphism of $S^{\#}$ may be extended 
to an automorphism of $S$. Therefore using the characterization of automorphisms of Croisot-Teissier semigroups in [2] we are able to describe the automorphisms of $S$ completely. The next definition was introduced in [2, p.228].

Definition 2 An automorphism $\phi$ of a semigroup of transformations $S$ is said to be range-preserving if for all $f, g \in S, R(f) \subseteq R(g)$ if and only if $R(\phi(f)) \subseteq R(\phi(g))$.

The following decomposition of the union $W$ of all well-separated sets, and the associated decomposition of the Croisot-Teissier semigroup into a union of its right ideals was first described in [2]. Here we present a brief account of these decompositions and some terminology introduced in [2], which we use to give a description of all rangepreserving automorphisms of $S^{\#}$ and automorphisms of $S$. Let $K$ be an index set containing $I$ such that $\mathcal{E}^{\#}=\left\{\mathcal{E}_{i} \mid i \in K\right\}$. A pair of $\mathcal{C}_{q}$ sets $A$ and $B$ are said to be $\sigma$-related if whenever $A$ and $B$ both meet a non-singleton class $[u]$ of $\delta=\cap\left\{\mathcal{E}_{i}: i \in K\right\}$ there exist $F_{1}=A, F_{2}, \ldots, F_{n}=B \in \mathcal{C}_{q}$ such that $F_{j} \cap F_{j+1} \in \mathcal{C}_{q}$ and $F_{j} \cap[u] \neq \Phi$. Let $\left\{\mathcal{M}_{\alpha} \mid \alpha \in \Omega\right\}$ be the collection of all maximal families of $\sigma$-related $\mathcal{C}_{q}$ sets. For each $\alpha \in \Omega$ let $\mathcal{A}_{\alpha}=\cup\left\{A \mid A \in \mathcal{M}_{\alpha}\right\}$ and $\mathcal{I}_{\alpha}=\left\{f \in S^{\#} \mid R(f) \in \mathcal{M}_{\alpha}\right\}$, a right ideal of $S^{\#}$. A set $\left\{h_{\alpha} \mid \alpha \in \Omega\right\}$ of permutations of $W$ is termed compatible if there exists a permutation $k$ of $W / \rho$ such that the equality of the $\rho$-classes $\left[h_{\alpha}(x)\right]=k([x])$ holds for all $\alpha \in \Omega$ and $x \in W, k$ induces a permutation of the set $\left\{\left(\left.\mathcal{E}_{i}\right|_{W \times W}\right) / \rho: i \in K\right\}$ of the equivalences on $W / \rho$, and $h_{\alpha} f=h_{\beta} f$ for all $f \in \mathcal{I}_{\alpha} \cap \mathcal{I}_{\beta}$. For each $\mathcal{E}_{i}$ define $\mathcal{B}\left(\mathcal{E}_{i}\right)=\left\{[x] \in \mathcal{E}_{i} \mid[x] \cap W=\Phi\right\}$ and let $J=\left\{i \in K \mid \mathcal{B}\left(\mathcal{E}_{i}\right) \neq \Phi\right\}$. The following result describing range-preserving automorphisms of a Croisot-Teissier semigroup was proved in [2, Theorem 4.4]. The statement is in terms of the maximal Croisot-Teissier subsemigroup $S^{\#}$ of $S$.

Proposition 3 Let $\phi^{\#}$ be a range-preserving automorphism of $S^{\#}$. There exists, uniquely,

(i) a compatible set $\left\{h_{\alpha} \mid \alpha \in \Omega\right\}$ of permutations of $W$,

(ii) a permutation $z^{\#}$ of $\mathcal{E}^{\#}$ such that $\left.z^{\#}\left(\mathcal{E}_{i}\right)\right|_{W}=h_{\alpha}\left(\left.\mathcal{E}_{i}\right|_{W}\right)$ for any $\mathcal{E}_{i} \in \mathcal{E}^{\#}$ and $\alpha \in \Omega$, and

(iii) a family of bijections $\left\{y_{i} \mid i \in J\right\}$ where $y_{i}: \mathcal{B}\left(\mathcal{E}_{i}\right) \rightarrow \mathcal{B}\left(z^{\#}\left(\mathcal{E}_{i}\right)\right)$, such that

1) $\left.\phi^{\#}(f)\right|_{W}=h_{\alpha} f h_{\alpha}^{-1}$ for all $f \in \mathcal{I}_{\alpha}$,

2) $\pi(f)=z^{\#}(\pi(f))$, and

3) $\phi^{\#}(f)(D)=h_{\alpha} f y_{i}^{-1}(D)$ for all $f \in \mathcal{I}_{\alpha}$ with $\pi(f)=\mathcal{E}_{i}$ and $D \in \mathcal{B}\left(z^{\#}\left(\mathcal{E}_{i}\right)\right)$.

Conversely, given $S^{\#}$ and (i), (ii), and (iii), there exists a unique range-preserving automorphism $\phi^{\#}$ of $S^{\#}$ such that 1), 2), and 3) hold. 
Definition 4 Given an automorphism $\phi^{\#}$ of $S^{\#}$ and an equivalence class $A$ of $\mathcal{E}_{i}$ let $\tilde{\mathcal{A}}$ be the equivalence class of $z^{\#}\left(\mathcal{E}_{i}\right)$ containing $h_{\alpha}(x)$, for some $\alpha \in \Omega$, if $x \in A \cap W \neq \Phi$, and $\tilde{\mathcal{A}}=y_{i}(A)$ if $A \cap W$ is empty. An automorphism $\phi^{\#}$ of $S^{\#}$ is said to be $r$ unionpreserving if whenever $\mathcal{E}_{i}, \mathcal{E}_{j} \in \mathcal{E}^{\#}$ with $\mathcal{E}_{i}^{(r)} \cap \mathcal{E}_{j}^{(r)} \neq \Phi$ and $\mathcal{C}, \mathcal{D}$ are collections of fewer than $r$ classes in $\mathcal{E}_{i}$ and $\mathcal{E}_{j}$ respectively, then $\cup \mathcal{C}=\cup \mathcal{D}$ if and only if $\cup\{\tilde{\mathcal{A}}: A \in$ $\mathcal{C}\}=\cup\{\tilde{\mathcal{B}}: B \in \mathcal{D}\}$.

Definition 5 An automorphism $\phi^{\#}$ of $S^{\#}$ is said to be $r$ glueing-preserving if for all $\mathcal{E}_{i} \in \mathcal{E}^{\#}, \mathcal{E}_{i} \in \mathcal{E}_{j}^{(r)}$ if and only if $z^{\#}\left(\mathcal{E}_{i}\right) \in z^{\#}\left(\mathcal{E}_{j}\right)^{(r)}$.

We are now ready to present the main result of the paper describing automorphisms of $S$. The proof of the theorem below is the content of Lemmas 7 to 16 and Propositions 8 and 17.

Theorem 6 An automorphism $\phi$ of $S$ induces a range-preserving, $r$ union-preserving, $r$ glueing-preserving automorphism $\phi^{\#}$ of $S^{\#}$. Conversely every range-preserving, $r$ union-preserving, $r$ glueing-preserving automorphism of $S^{\#}$ can be extended uniquely to an automorphism of $S$.

Lemma 7 Let $f, g \in S$. Then

(i) $\pi(f) \in \pi(g)^{(r)}$ if and only if $f \in S^{1} g$;

(ii) $\pi(f)=\pi(g)$ if and only if $S^{1} f=S^{1} g$;

(iii) $f \mathcal{L} g$ if and only if $\pi(f)=\pi(g)$.

Proof. Observe that (ii) follows directly from (i), while to prove (i) it suffices to show that if $\pi(f) \in \pi(g)^{(r)}$ then $f \in S^{1} g$. For this choose any $\mathcal{E}_{i} \in \mathcal{E}^{\#}$ and let $\mathcal{D}$ be the set of all classes in $\mathcal{E}_{i}$ that have a non-empty intersection with $R(g)$. Define an equivalence relation $\mu$ on the classes of $\mathcal{D}$ via $(A, B) \in \mu$ if and only if $f g^{-1}(A)=f g^{-1}(B)$. Since $\pi(g) \in \mathcal{E}^{(r)}$ and $\pi(f) \in \pi(g)^{(r)}$, it follows that there are fewer than $r$ classes of $\mathcal{D}$ in each $\mu$-equivalence class. Let $\eta: \mathcal{E}_{i}-\mathcal{D} \rightarrow \mathcal{D}$ be a one-to-one mapping (it is readily checked that $\left|\mathcal{E}_{i}-\mathcal{D}\right| \leq|\mathcal{D}|=p$ ). Extend $\mu$ to $\mathcal{E}_{i}$ by adjoining to each $\mu$-equivalence class the preimages of its elements under $\eta$. Fewer than $r$ classes are adjoined, since $\eta$ is one-to-one. The equivalence classes of $\mu$ on $\mathcal{E}_{i}$ naturally provide us with an $r$ glueing $\mathcal{P}$ of $\mathcal{E}_{i}$. Note that $R(g)$ contains a transversal of $\mathcal{P}$ and let $t$ be a transformation of $X$ having $\pi(t)=\mathcal{P}$ and for every $y=g(x), t(y)=f(x)$. Then $t \in S$ and $f=t g \in S^{1} g$, as required. Finally note that (iii) is a restatement of (ii).

Let $\phi$ be an automorphism of $S$. The following is a consequence of Lemma 7 and the definition of $S^{\#}$. 
Proposition 8 1. The correspondence $z: \mathcal{E}^{(r)} \rightarrow \mathcal{E}^{(r)}$ defined by $z(\pi(f))=\pi(\phi(f))$ is a bijection such that $\mathcal{P} \in \mathcal{E}_{i}^{(r)}$ if and only if $z(\mathcal{P}) \in z\left(\mathcal{E}_{i}\right)^{(r)}$.

2. The restriction $\phi^{\#}$ of $\phi$ to $S^{\#}$ is an $r$ glueing-preserving automorphism of $S^{\#}$.

Lemma 9 For every $A \in \mathcal{C}_{q}$ and $\mathcal{E}_{i} \in \mathcal{E}$ there exists a $\mathcal{P} \in \mathcal{E}_{i}^{(r)}$ such that $A$ is a total transversal of $\mathcal{P}$.

Proof. Note that $A$ is a partial transversal of $\mathcal{E}_{i}$ and let $\mathcal{D}$ be the set of all classes in $\mathcal{E}_{i}$ that have an empty intersection with $A$. Then $|\mathcal{D}| \leq p$, and there exists a one-to-one function $\eta: \mathcal{D} \rightarrow A$. Let $\mathcal{P}$ be a partition of $X$ consisting of all classes of $\mathcal{E}_{i}$ that do not intersect $\eta(\mathcal{D})$ and all the sets of the form $F \cup[\eta(F)]$, where $[\eta(F)]$ is the $\mathcal{E}_{i}$-class of $\eta(F)$ and $F \in \mathcal{D}$. Then $\mathcal{P} \in \mathcal{E}_{i}^{(r)}$ as required.

Lemma 10 For every $A \in \mathcal{C}_{q}$ and $\mathcal{E}_{i} \in \mathcal{E}$ there exists an idempotent $e$ in $S$ with $R(e)=A$ and $\pi(e) \in \mathcal{E}_{i}^{(r)}$.

Proof. Using Lemma 9 choose $\mathcal{P} \in \mathcal{E}_{i}^{(r)}$ such that $A$ is a total transversal of $\mathcal{P}$. Then the required idempotent is a transformation $e$ of $X$ with $\pi(e)=\mathcal{P}, R(e)=A$ and $e(a)=a$, for every $a \in A$.

\section{Proposition $11 \quad S^{2}=S$}

Proof. For an $f$ in $S$ let $e$ be an idempotent in $S$ with $R(e)=R(f)$ (Lemma 10). Then $f=e f \in S^{2}$.

Lemma 12 (i) For $f$ and $g$ in $S, R(f) \subseteq R(g)$ if and only if for every idempotent $e$ in $S$, eg $=g$ implies ef $=f$.

(ii) All automorphisms of $S$ are range-preserving.

Proof. Observe that (ii) is an easy consequence of (i) and the fact that idempotents are preserved under automorphisms. To prove (i) note that if $R(f) \subseteq R(g)$ and $e$ is an idempotent such that $e g=g$ then $e$ is the identity on $R(g)$, hence $e$ is the identity on $R(f)$, and so $e f=f$. Conversely assume $x \in R(f)-R(g)$ and let $x=f(y)$. Choose an idempotent $e$ in $S$ with $R(e)=R(g)$. Then $e g=g$ while $e f(y)=e(x) \neq x=f(y)$, so that ef $\neq f$.

Note that the above result implies that the restriction $\phi^{\#}$ of $\phi$ to $S^{\#}$ is a rangepreserving automorphism of $S^{\#}$, described in Proposition 3. We will use it to describe $\phi$ itself.

Lemma 13 Let $f \in S$ with $R(f) \in \mathcal{M}_{\alpha}$, and take $x \in W$. Then $\phi(f)(x)=h_{\alpha} f h_{\alpha}^{-1}(x)$. 
Proof. We show that there exists a $g \in S^{\#}$ such that $f g \in S^{\#}$ and $x \in R(\phi(f))$. Let $[x]$ be the $\delta$-class containing $x$ and $V=h_{\alpha}^{-1}([x])$. Choose $A \in \mathcal{C}_{q}$ such that $A \cap V$ is non-empty and let $A \cap V=\{y\}$. Assume $\pi(f) \in \mathcal{E}_{i}^{(r)}$. Since $A$ is a partial transversal of $\mathcal{E}_{i}$ and each class of $\pi(f)$ consists of fewer than $r$ classes of $\mathcal{E}_{i}, r \leq p$, there exists a subset $D$ of $A$ of cardinality $p$ such that $y \in D$ and $D$ is a partial transversal of $\pi(f)$. Let $D \in \mathcal{M}_{\beta}$, for some $\beta \in \Omega$, and $h_{\beta}^{-1}(x)=w$. Note that $h_{\beta}^{-1}([x])=h_{\alpha}^{-1}([x])$, so that $y \delta w$ (see [2, p.211] for details), and choose $g \in S^{\#}$ with $R(g)=(D-\{y\}) \cup\{w\}$ and $g(v)=w$ for some $v \in W$. Then $g \in \mathcal{I}_{\beta}$, and since $\pi(g)=\pi(f g)$ and $R(f g) \subseteq R(f)$ we have that $f g \in S^{\#} \cap \mathcal{I}_{\alpha}$. Let $u=h_{\beta}(v)$, then $u \in W$ and $\phi(g)(u)=h_{\beta} g h_{\beta}^{-1}(u)=h_{\beta} g h_{\beta}^{-1} h_{\beta}(v)=$ $h_{\beta} g(v)=h_{\beta}(w)=x ; \quad \phi(f g)(u)=h_{\alpha} f g h_{\alpha}^{-1}(u) ; \phi(f)(x)=\phi(f) \phi(g)(u)=\phi(f g)(u)=$ $h_{\alpha} f g h_{\alpha}^{-1}(u)=h_{\alpha} f g h_{\alpha}^{-1} h_{\beta}(v)=h_{\alpha} f g(v)=h_{\alpha} f(w)=h_{\alpha} f h_{\beta}^{-1}(x)=h_{\alpha} f h_{\alpha}^{-1}(x)$, since $h_{\alpha}^{-1} h_{\beta}(v)$ and $v, h_{\beta}^{-1}(x)$ and $h_{\alpha}^{-1}(x)$ are pairwise $\delta$-related.

Recall (Proposition 8) that $\phi$ induces a permutation $z: \mathcal{E}^{(r)} \rightarrow \mathcal{E}^{(r)}$ defined by $z(\pi(f))=\pi(\phi(f))$.

Lemma 14 If $\mathcal{P} \in \mathcal{E}_{i}^{(r)}$ and $C$ and $D$ are classes of $\mathcal{E}_{i}$, then $C \cup D$ is a subset of a $\mathcal{P}$-class if and only if $C \cup D$ is a subset of a class of $z(\mathcal{P})$.

Proof. Let $f \in S$ with $\pi(f)=\mathcal{P}$. Using Lemma 7 choose $g, t \in S$, such that $f=\operatorname{tg}$ and $\pi(g)=\mathcal{E}_{i}$. Assume $R(t) \in \mathcal{M}_{\alpha}$ (and so $\left.R(f) \in \mathcal{M}_{\alpha}\right), R(g) \in \mathcal{M}_{\beta}$. If $C \in \mathcal{B}\left(\mathcal{E}_{i}\right)$ then $\tilde{C}=y_{i}(C)$, and by Proposition $3, \phi(f)(\tilde{C})=\phi(f)\left(y_{i}(C)\right)=\phi(t) h_{\beta} g y_{i}^{-1}\left(y_{i}(C)\right)=$ $h_{\alpha} t h_{\alpha}^{-1} h_{\beta} g(C)=h_{\alpha} f(C)$, by Lemma 13 , since $h_{\beta} g(C) \in W$. If $D \in \mathcal{B}\left(\mathcal{E}_{i}\right)$ then $\phi(f)(\tilde{C})=\phi(f)(\tilde{D})$ iff $\phi(f)\left(y_{i}(C)\right)=\phi(f)\left(y_{i}(D)\right)$ iff $f(C)=f(D)$, as required. If $D$ is not in $\mathcal{B}\left(\mathcal{E}_{i}\right)$, then there is an $x \in D \cap W$ and $\phi(f)(\tilde{D})=\phi(f)\left(h_{\alpha}(x)\right)=h_{\alpha} f h_{\alpha}^{-1} h_{\alpha}(x)=$ $h_{\alpha} f(D)$, so again $\phi(f)(\tilde{C})=\phi(f)(\tilde{D})$ iff $f(C)=f(D)$. The remaining case when $C$ is not in $\mathcal{B}\left(\mathcal{E}_{i}\right)$ can be dealt with in a similar manner.

Corollary 15 The automorphism $\phi^{\#}$ is $r$ union-preserving.

Lemma 16 Let $f \in S$ with $R(f) \in \mathcal{M}_{\alpha}, \pi(f) \in \mathcal{E}_{i}^{(r)}$, and $D \in z(\pi(f))$ with $D \cap W=\Phi$. Then $\phi(f)(D)=h_{\alpha} f y_{i}^{-1}(C)$, where $C \subseteq D, C \in z\left(\mathcal{E}_{i}\right)$ and $C \cap W=\Phi$.

Proof. Choose $g, t \in S$, with $\pi(g)=\mathcal{E}_{i}$ such that $f=t g$. Assume $R(g) \in \mathcal{M}_{\beta}, R(t) \in$ $\mathcal{M}_{\tau}$. Since $D \in z(\pi(f)) \in z\left(\mathcal{E}_{i}^{(r)}\right)$, there exists a subset $C$ of $D, C \in z\left(\mathcal{E}_{i}\right)$. Then $\phi(f)(D)=\phi(f)(C)=\phi(t) \phi(g)(C)=h_{\tau} t h_{\tau}^{-1} h_{\beta} g y_{i}^{-1}(C)$, since $g \in S^{\#}$, and $h_{\beta} g y_{i}^{-1}(C) \in$ $W$. Since for any $a \in X, h_{\tau}^{-1} h_{\beta} g(a)$ and $g(a)$ are $\mathcal{E}_{j}$-equivalent for any $j, h_{\tau} t h_{\tau}^{-1} h_{\beta} g y_{i}^{-1}(C)$ $=h_{\tau} t g y_{i}^{-1}(C)=h_{\tau} f y_{i}^{-1}(C)=h_{\alpha} f y_{i}^{-1}(C)$, because $R(f)$ is a subset of $R(t)$.

Proposition 17 Let $\mu$ be a range-preserving, $r$ union-preserving and $r$ glueing-preserving automorphism of $S^{\#}$. Then $\mu$ can be extended uniquely to an automorphism $\tau$ of $S$. 
Proof. Let $\mu$ be as stated, and $\left\{h_{\alpha} \mid \alpha \in \Omega\right\}, z^{\#},\left\{y_{i} \mid i \in I\right\}$ be the parameters describing $\mu$ as in Proposition 3. We extend $z^{\#}$ to a permutation $z$ of $\mathcal{E}^{(r)}$ as follows. Define a mapping $z$ from $\mathcal{E}^{(r)}$ to itself such that $z\left(\mathcal{E}_{i}\right)=z^{\#}\left(\mathcal{E}_{i}\right), i \in K$, and for $\mathcal{P} \in$ $\mathcal{E}_{i}^{(r)}, z(\mathcal{P}) \in\left(z^{\#}\left(\mathcal{E}_{i}\right)\right)^{(r)}$ such that $\tilde{B} \cup \tilde{C}$ is a subset of a $z(\mathcal{P})$-class if and only if $B \cup C$ is a subset of a $\mathcal{P}$-class. To see that $z$ is well-defined assume $\mathcal{P} \in \mathcal{E}_{i}^{(r)} \cap \mathcal{E}_{j}^{(r)}$, and let $F$ be a $\mathcal{P}$-class such that $F=\cup\left\{G \mid G \in \mathcal{E}_{i}\right\}=\cup\left\{H \mid H \in \mathcal{E}_{j}\right\}$. Since $F$ is a union of fewer than $r$ classes of $\mathcal{E}_{i}$ or $\mathcal{E}_{j}$ and $\mu$ is $r$ union-preserving, we have that $\cup\left\{\tilde{G} \mid G \in \mathcal{E}_{i}\right\}=\cup\left\{\tilde{H}: H \in \mathcal{E}_{j}\right\}$, as required.

Define a mapping $\tau$ on $S$ as follows. For $f \in S^{\#}$, let $\tau(f)=\mu(f)$. For $f \in S$ with $R(f) \in \mathcal{M}_{\alpha}$ and $\pi(f) \in \mathcal{E}_{i}^{(r)}$, let $\pi(\tau(f))=z(\pi(f))$, and $\tau(f)(x)=h_{\alpha} f h_{\alpha}^{-1}(x)$ if $x \in W$, while for an $[x] \in \mathcal{B}\left(z\left(\mathcal{E}_{i}\right)\right), \tau(f)(x)=h_{\alpha} f y_{i}^{-1}(D)$, where $D \subseteq[x], D \in \mathcal{E}_{i}$.

To see that $\tau(f)$ is a mapping assume that $[x] \in \mathcal{B}\left(z\left(\mathcal{E}_{i}\right)\right)$ and there exists $u \in$ $W, u \in C \in z\left(\mathcal{E}_{i}\right)$ such that $x, u$ are in the same class of $\pi(\tau(f))$. Then $\tau(f)(x)=$ $h_{\alpha} f y_{i}^{-1}(x), \tau(f)(u)=h_{\alpha} f h_{\alpha}^{-1}(u)$, and since by the definition of $z, f h_{\alpha}^{-1}(C)=f y_{i}^{-1}(x)$ we have that $h_{\alpha} f y_{i}^{-1}(x)=h_{\alpha} f h_{\alpha}^{-1}(u)$, as required. The proof that $\tau$ is a homomorphism is analogous to that of Proposition 3 (see $[2, \S 4]$ ).

We now turn to the description of the Green's relations on $S$. Just as the maximal Croisot-Teissier subsemigroup $S^{\#}=\{f \in S \mid \pi(f t)=\pi(t)$ for all $t \in S\}$ of $S$ played a crucial role in the description of the automorphisms of $S$, so the maximal regular subsemigroup of $S$ aids in the description of the Green's relations on $S$. Let $E(S)$ be the set of all idempotents of $S$, and define

$$
N=\{f \in S \mid R(f t)=R(f) \text { for some } t \in S\} .
$$

Then $N$ is a subsemigroup of $S$ containing $E(S)$. Moreover $N$ contains all the regular transformations in $S$, for if $f$ is regular then $f g f=f$, for some $g \in S$, and $R(f(g f))=$ $R(f)$. We show in Proposition 20 that $N$ is the maximal regular subsemigroup of $S$.

Proposition 18 For distinct $f, g \in S, f \mathcal{R} g$ iff $f, g \in N$ with $R(f)=R(g)$.

Proof. Assume $f \mathcal{R} g$, then $f s=g$ and $g t=f$, for some $s, t \in S$. Therefore $R(f)=R(g)$, and so $R(f)=R(g)=R(f s)=R(g t)$, hence $f, g \in N$. Conversely, assume $f, g \in N$ with $R(f)=R(g)$. Then there exist $A, B \in C_{q}$ such that $A$ and $B$ are transversals of $\pi(f)$ and $\pi(g)$ respectively. Let $h: B \rightarrow A$ be a bijection such that $h(b)=\left\{f^{-1} g(b)\right\} \cap A$, for each $b \in B$. Define $s \in S$ such that $R(s)=A, \pi(s)=\pi(g)$, and for each $b \in B$, a transversal of $\pi(s), s(b)=h(b)$. Then $f s=g$, and a transformation $t \in S$ such that $g t=f$ may be constructed similarly.

Proposition 19 For distinct $f, g \in S, f \mathcal{D} g$ iff either $\pi(f)=\pi(g)$, or $f, g \in N$.

Proof. Assume $f \mathcal{D} g$, so that $f \mathcal{L} s$ and $s \mathcal{R} g$, for some $s \in S$. Then $\pi(f)=\pi(s)$ (Lemma 7) and if $s \neq g$, then $s, g \in N$ so that $f \in N$ also. Conversely, if $f, g \in N$, 
choose $s \in S$ with $R(s)=R(f)$ and $\pi(s)=\pi(g)$. Then $s \in N$ and $f \mathcal{L} s \mathcal{R} g$.

Since $N$ consists of precisely those elements $f$ of $S$ whose partition $\pi(f)$ has a total transversal amongst $\mathcal{C}_{q}$ sets, $N$ is a $\mathcal{D}$-class of $S$. Moreover $N$ is a $\mathcal{D}$-class of $S$ containing the set of idempotents of $S$, so that every element of $N$ is regular. This, in conjunction with the earlier observation that $N$ contains all the regular elements of $S$, proves the next result.

Proposition $20 \mathrm{~N}$ is the maximal regular subsemigroup of $S$.

Proposition $21 S$ is simple.

Proof. Since $N$ is a $\mathcal{D}$-class of $S$ (see the remark after Proposition 19) and $\mathcal{D} \subseteq \mathcal{J}$, it suffices to show that for any $f \in S$ there exists $g \in N$ such that $f \mathcal{J} g$. A proof similar to that of Lemma 9 yields that for an $f \in S$ there exists $\mathcal{P} \in(\pi(f))^{(r)}$ such that for $g \in S$ with $\mathcal{P}=\pi(g)$, we have that $g \in N$. Now let $i$ be such that $\pi(f) \in \mathcal{E}_{i}^{(r)}$, and choose $\mathcal{Q} \in \mathcal{E}_{i}^{(r)}$ such that for $A, B \in \mathcal{E}_{i}, A$ and $B$ are in the same class of $\mathcal{Q}$ if and only if both $A \cap R(f)$ and $B \cap R(g)$ are non-empty, and $f^{-1}(A)$ and $f^{-1}(B)$ are in the same class of $\mathcal{P}$. Then for $s \in S$ with $\pi(s)=\mathcal{Q}$ we have that $\pi(s f)=\mathcal{P}$, and so $s f \in N$. Let $s f=g$. We show that there exist $u, v \in S$ such that $f=u g v$. Let $v$ be such that $R(v)$ is a transversal of $\pi(g)$ and $\pi(v)=\pi(f)$. Then $R(g v)=R(g)$ and $\pi(g v)=\pi(f)$. Choose a bijection $w$ from $R(g)$ onto $R(f)$ such that $w(g v(x))=f(x)$ for all $x \in X$. Let $u \in N$ be such that $R(g)$ is a transversal of $\pi(u)$, and for each $y \in R(g), u(y)=w(y)$. Then $f=u g v$, as required.

\section{References}

[1] Clifford, A.H. and G.B. Preston, The Algebraic Theory of Semigroups, II, American Mathematical Society, 1967.

[2] Levi, Inessa, K.C. O'Meara, and G.R. Wood, Automorphisms of Croisot-Teissier Semigroups, Journal of Algebra, 101(1), 1986, 190-245.

[3] Levi, Inessa, Green's Relations on Croisot-Teissier Semigroups, Semigroup Forum, $33,1986,299-307$.

[4] Levi, Inessa and Steven Seif, Range Sets and Partition Sets in Connection with Congruences and Algebraic Invariants, Semigroup Forum, 1993, to appear.

[5] Lindsey, D. and B. Madison, Congruences on Croisot-Teissier Semigroups, Proceedings of the Conference on Semigroups in honor of Alfred H. Clifford, Tulane Univ., New Orleans, La., 1979, 193-206. 
[6] Mielke, B.W., Regular Congruences on Croisot-Teissier and Baer-Levi Semigroups, J. Math. Soc. Japan, 24, 1972, 539-551.

[7] Mielke, B.W., Regular Congruences on a Simple Semigroup with a Minimal Right Ideal, Publ. Math. Debrecen, 20, 1973, 79-84.

[8] Mielke, B.W., Completely Simple Congruences on Croisot-Teissier Semigroups, Semigroup Forum, 9, 1975, 283-293.

Mathematics Department

University of Louisville

Louisville, KY 40292

U.S.A.
Mathematics Department

University of Canterbury

Christchurch, 1

New Zealand 